Pacific Journal of Mathematics

GROUPS OF KNOTS IN HOMOLOGY 3-SPHERES THAT ARE 


\section{GROUPS OF KNOTS IN HOMOLOGY 3-SPHERES THAT ARE NOT CLASSICAL KNOT GROUPS}

\section{MAGNHILD LIEN}

In this paper we attempt to enlarge classical knot groups $K$ by adding a root to a meridian of $K$. Thus if $K$ is a classical knot group with a meridian $\mu$, then the groups we study are of the form $G=K_{\mu=t^{q}}^{*}\langle t\rangle$. This group can always be realized as the group of a knotted 3-sphere in $S^{5}$. By using explicit geometric constructions we also show that such a group $G$ is a 2-knot group and the group of a knot in a homology 3-sphere. Finally, we show that $G$ is not realizable by any knot in $S^{3}$.

1. Introduction. In $\left[\mathbf{R}_{1}\right] \mathrm{J}$. Ratcliffe gave an example of a group $\Gamma$ that is the group of a fibered knot in a homology 3-sphere which cannot be realized as the group of a classical knot. Let $K$ be the group of the trefoil knot and let $\mu \in K$ represent a meridian. As seen in $\left[\mathbf{R}_{1}\right] \Gamma$ can be expressed as a free product with amalgamation $K *\langle t\rangle$.

In this paper we generalize the result in $\left[\mathbf{R}_{1}^{\mu=t^{2}}\right.$. We study groups $G$ obtained from classical knot groups $K$ by forming an amalgamated free product of $K$ with $Z$. More specifically if $K$ is a classical knot group with meridian $\mu$, then $G=K \underset{\mu=t^{q}}{*}\langle t\rangle$. Note that if $q=1$ then $G=K$. Hence we assume $q>1$. One natural question to ask about $G$ is if $G$ is the group of a knot in any dimension. We show that $G$ is the group of a knotted 2-sphere in $S^{4}$. Furthermore we show that $G$ can be realized as the group of a knot in a homology 3-sphere. However, $G$ is not a classical knot group.

I would like to thank the referee for his suggestion of how to construct the 2-sphere in $\$ 3$. This made the third section a lot simpler than it was in the first version of this paper.

2. Preliminaries. In this paper we work in the smooth category. $S^{n}$ and $B^{n}$ denote the standard $n$-sphere and $n$-ball. If $N$ and $M$ are manifolds and $f: M \rightarrow N$ is a map then both of the induced homomorphisms $\pi_{1}(M) \rightarrow \pi_{1}(N)$ or $H_{1}(M) \rightarrow H_{1}(N)$ will be denoted by $f_{*}$. Homeomorphism between spaces and isomorphism between groups are denoted by $\cong$. An $n$-dimensional knot is the image of a smooth 
embedding $\Sigma^{n}$ of $S^{n}$ into $S^{n+2}$ or $R^{n+2}$. By the knot group we mean $\pi_{1}\left(S^{n+2}-\Sigma^{n}\right)$. For $n=1$, we call these groups classical knot groups.

We define the deficiency of a group presentation with $n$ generators and $m$ relators to be the integer $n-m$. The following well known proposition is due to Kervaire [K].

Proposition 2.1. If a group $K$ has a deficiency one presentation and $K / K^{\prime} \cong Z$ then $H_{2}(K)=0$.

Consider an oriented knot $\Sigma$ in $S^{3}$. Remove an open neighbourhood $N$ of $\Sigma$ in $S^{3}$ to produce the knot manifold $X=S^{3}-N$. The preferred meridian, longitude pair $(\mu, \lambda)$ of $\Sigma$ are two nontrivial simple closed curves on $\operatorname{Bd}(X)$ such that $\mu$ bounds a disk in $N$ and $\lambda$ is homologically trivial in $X$.

Definition 2.2. A $(p, q)$-curve is a simple closed curve $J$ on $\operatorname{Bd}(X)$ that is homotopic to $\mu^{p} \lambda^{q}$ where $p$ and $q$ are relatively prime. We also call $J$ a $(p, q)$-cable about $\Sigma$.

3. A 2-knot with group $K \underset{\mu=t^{q}}{*}\langle t\rangle$. Let $K$ be a classical knot group with meridian $\mu$. We construct a new group $G$ by adding a $q$ th root (via amalgamted free product) to the meridian $\mu$ of $K$, i.e., $G=K_{\mu=t^{q}}^{*}\langle t\rangle$. The following proposition is easy to verify using Kervaire's characterization of high dimensional knot groups ([K], Theorem 1$)$.

\section{Proposition 3.1. The group $G$ is a high dimensional knot group.}

Proof. Since $K$ is a classical knot group it has a deficiency one presentation. We can thus obtain a deficiency one presentation of $G$ from a presentation of $K$ by adding one more generator $(t)$ and one more relation $\left(\mu=t^{q}\right)$. Moreover since $K$ is a knot group and hence satisfies the conditions of Kervaire's characterization it is straightforward to check that $G / G^{\prime} \cong Z$ and that $G /\langle\langle t\rangle\rangle=1$. By Proposition 2.1 we obtain $H_{2}(G)=0$, and it follows that $G$ can be realized as the group of a knotted 3-sphere in $S^{5}$.

Let $\Sigma$ be a knot in $S^{3}$ with group $K$. We shall construct a knotted 2-sphere $\Sigma^{4}$ with $\pi_{1}\left(S^{4}-\Sigma^{2}\right)=K_{\mu=t^{q}}^{*}\langle t\rangle$. The equatorial cross-section of this 2 -sphere will be a $(1, q)$-cable about the composite knot $\Sigma \#-\Sigma *$, where $-\Sigma^{*}$ is the mirror image of $\Sigma$ with its orientation reversed. 
Definition 3.2. A knot $\Sigma$ in $S^{3}$ is a slice knot if there exists a smooth disk $D$ in $B^{4}$ such that $\operatorname{Bd}(D)=\Sigma$.

THEOREM 3.3. Let $\Sigma$ be a knot in $S^{3}$ and let $L$ be the $(1, q)$-cable about $\Sigma \#-\Sigma^{*}$. Then L is a slice knot.

Proof. For any knot $\Sigma$, the knot $\Sigma \#-\Sigma^{*}$ is a slice knot [F, M]. To construct a slice disk $D$ in $B^{4}$ with $\operatorname{Bd}(D)=\Sigma \#-\Sigma^{*}$, we do as follows. First note that $\left(S^{3}, \Sigma\right)=\left(B^{3}, \beta\right) \cup_{\partial}\left(B^{3}, B^{1}\right)$ where $\beta$ is a knotted arc and $\left(B^{3}, B^{1}\right)$ is a standard ball pair. Remove the ball pair $\left(B^{3}, B^{1}\right)$ from $\left(S^{3}, \Sigma\right)$ and cross $\left(B^{3}, \beta\right)$ with the interval to obtain a disk $D=\beta \times I$ contained in $B^{4}$. Then $\operatorname{Bd}(D)=\Sigma \#-\Sigma^{*}$. Thus $D$ is the desired disk. Let $N=D \times$ int $B^{2}$ be an open neighbourhood of the slice disk and let $M=B^{4}-N$. Then $D \times \operatorname{Bd}\left(B^{2}\right)$ is in $\operatorname{Bd}(M)$. We shall attach a 2-handle $B^{2} \times B^{2}$ which contains a slice disk for the trivial $(1, q)$-torus knot to $M$ along $D \times \operatorname{Bd}\left(B^{2}\right)$. If $B^{2} \times B^{2}$ is attached along $S^{1} \times B^{2}$ then let the torus knot be the $(1, q)$-cable about the core of the solid torus $B^{2} \times S^{1}$. Note that the attaching sphere $S^{1} \times\{0\}$ represents the $q$ th power of the meridian of the torus knot. Since the image of $\{*\} \times S^{1}$ under the attaching map is $\operatorname{Bd}(D)=\Sigma \#-\Sigma^{*}$ it follows that the image of the boundary of the slice disk for the $(1, q)$-torus knot is $L$. Thus there exists a disk in $B^{4}$ with boundary $L$.

Since $L$ is a slice knot, we can use $L$ as the equatorial section of a knotted 2-sphere in $S^{4}$ by joining together smooth disks in $B_{+}^{4}$ and $B_{-}^{4}$ bounded by $L$. We denote the 2 -sphere obtained this way by $S(\Sigma, q)$.

THEOREM 3.4. Let $\Sigma$ be a knot in $S^{3}$ with group $K$ and meridian $\mu$, $q>1$ and let $S(\Sigma, q)$ be the 2-knot described above. Then

$$
\pi_{1}\left(S^{4}-S(\Sigma, q)\right)=K \underset{\mu=t^{q}}{*}\langle q\rangle .
$$

Proof. If $\tilde{D}$ is the slice disk for the $(1, q)$-cable about $\Sigma \#-\Sigma^{*}$ it suffices to show that $\pi_{1}\left(B^{4}-\tilde{D}\right)=K \underset{\mu=t^{q}}{*}\langle t\rangle$. Using the notation from the proof of Theorem 3.3 we have that

$$
B^{4}-\tilde{D}=M \cup_{S^{1} \times B^{2}-\operatorname{Bd}(\tilde{D})}\left(B^{2} \times B^{2}\right)-\tilde{D} .
$$

Since $M$ is homotopic to $B^{4}-D$ which equals $\left(B^{3}-\beta\right) \times I$ it follows that $\pi_{1}(M)=\pi_{1}\left(B^{3}-\beta\right)=K$. Moreover, since $\operatorname{Bd}\left(\tilde{D}_{1}\right)$ is the (trivial) $(1, q)$-torus knot we get that the fundamental group of $B^{2} \times B^{2}-\tilde{D}$ is 
infinite cyclic generated by the meridian of the torus knot. Thus the fundamental group of $B^{4}-\tilde{D}$, is obtained from $\pi_{1}(M)$ by attaching a $q$ th root of the original meridian $\mu$, i.e. $\pi_{1}\left(B^{4}-\tilde{D}\right)=K \underset{\mu=t^{q}}{*}\langle t\rangle$.

4. 3-manifolds that can realize the group $K_{\mu=t^{q}}^{*}\langle t\rangle$. We now consider how close $G$ is to being a classical knot group. As the following shows the Alexander polynomial $\Delta_{G}(t)$ for $G$ is symmetric and it satisfies $\Delta_{G}(1)= \pm 1$.

TheOREM 4.1. Let $K$ be a classical knot group with Alexander polynomial $\Delta_{K}(t)$ and let $G=K_{\mu=t^{q}}^{*}\langle t\rangle$. Then the Alexander polynomial $\Delta_{G}(t)$ for $G$ satisfies $\Delta_{G}(t)=\Delta_{K}^{\mu=t^{q}}\left(t^{q}\right)$.

Proof. Let $K=\left\langle x_{0}, x_{1}, \ldots, x_{n} ; R_{1}, \ldots, R_{n}\right\rangle$ be a standard Wirtinger presentation for $K$ and let $A=\left[\partial R_{i} / \partial x_{j}\right]$ be the $n \times(n+1)$ Alexander matrix with respect to this presentation.

$$
A \text { is equivalent to } B=\left[\begin{array}{cccc}
\frac{\partial R_{1}}{\partial X_{0}} & \cdots & \frac{\partial R_{1}}{\partial X_{n-1}} & 0 \\
\frac{\partial R_{n}}{\partial X_{0}} & \cdots & \frac{\partial R_{n}}{\partial X_{n-1}} & 0
\end{array}\right]
$$

which is obtained from $A$ by adding the first $n$ columns to the last column. The Alexander polynomial for $K, \Delta_{K}(t)$ is the generator of the principal ideal generated by the determinants of all the $n \times n$ submatrices of $B$. Thus $\Delta_{K}(t)=$ determinant of the $n \times n$ submatrix obtained from $B$ by deleting the last column. The group $G$ has a presentation $\left\langle t, x_{0}, \ldots, x_{n}\right.$; $\left.R_{1}, \ldots, R_{n}, x_{0} t^{-q}\right\rangle$ and its Alexander matrix is

$$
\left[\begin{array}{ccccc} 
& & & & \\
& & A\left(t^{q}\right) & & 0 \\
1 & 0 & \cdots & 0 & k(t)
\end{array}\right]
$$

where $k(t)=\partial\left(x_{0} t^{-q}\right) / \partial t$. This matrix is equivalent to

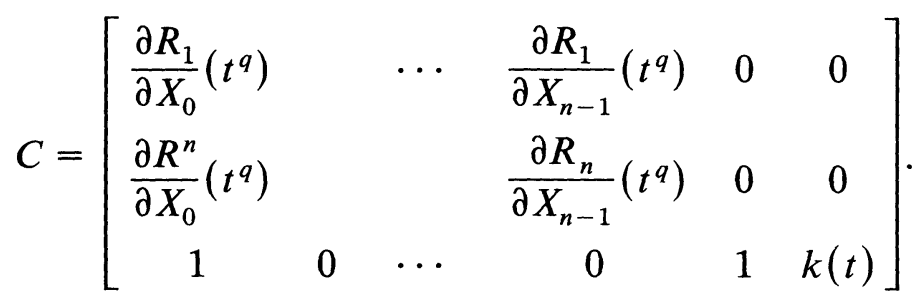


The ideal generated by all $(n+1) \times(n+1)$ minors of $C$ is easily seen to be principal and its generator is $\Delta_{K}\left(t^{q}\right)$. Hence $G$ has an Alexander polynomial $\Delta_{G}(t)$ and moreover $\Delta_{G}(t)=\Delta_{K}\left(t^{q}\right)$.

Since $K$ is a classical knot group there is a knot $\Sigma$ in $S^{3}$ with knot manifold $X$ such that $\pi_{1}(X)=K$. Let $(\mu, \lambda)$ be the preferred meridian, longitude pair for $\operatorname{Bd}(X)$, and let $A=\mu \times I$ be an annulus on $\operatorname{Bd}(X)$. By $T^{3}$ we mean the standard solid torus $S^{1} \times D^{2}$ in $R^{3}$. Furthermore, let $J$ be a $(1, q)$-curve on the boundary of $T^{3}$ and let $B=J \times I$ be an annulus on $\operatorname{Bd}\left(T^{3}\right)$. We construct a cabled 3-manifold $M[\mathbf{J}, \mathbf{M}]$ by glueing together $X$ and $T^{3}$ along the two annuli $A$ and $B$, i.e. $M=X \cup_{A=B} T^{3}$.

Proposition 4.2. $\pi_{1}(M)=K \underset{\mu=t^{q}}{*}\langle t\rangle$.

Proof. Let $\pi_{1}\left(T^{3}\right)=\langle t\rangle, \pi_{1}(A)=\langle\mu\rangle$ and $\pi_{1}(B)=\langle j\rangle$. The image of $\mu$ in $\pi_{1}(X)$ under the homomorphism induced by the inclusion map $A \rightarrow M$ is $\mu$, and the image of $j$ in $\pi_{1}\left(T^{3}\right)$ under the homomorphism induced by the inclusion map $B \rightarrow T^{3}$ is $t^{q}$. Thus by the Van-Kampen Theorem we conclude that $\pi_{1}(M)=K \underset{\mu=t^{q}}{*}\langle t\rangle$.

The boundary of $M$ is homeomorphic to $S^{1} \times S^{1}$, and if $(\mu, \lambda)$ is a standard meridian, longitude pair for $\operatorname{Bd}(X)$, then a basis for $\operatorname{Bd}(M)$ is $\mu, \lambda t^{-1}$.

THEOREM 4.3. If $K$ is a classical knot group with meridian $\mu$, then $G=K \underset{\mu=t^{q}}{*}\langle t\rangle$ is the group of a knot in a homology 3-sphere.

Proof. We construct a 3-manifold $M^{\prime}$ by sewing a solid torus to $M$ in such a way that a meridian of the solid torus is identified with the curve $\lambda t^{-1}$. Then $\pi_{1}\left(M^{\prime}\right)$ is obtained from $\pi_{1}(M)$ by adding the relation $t=\lambda$. Since $\pi_{1}(M)=K \underset{\mu=t^{q}}{*}\langle t\rangle$ we get that $\pi_{1}\left(M^{\prime}\right)=K /\left\langle\left\langle\mu \lambda^{-q}\right\rangle\right\rangle$. It is now easy to see that $\stackrel{\mu}{H}_{1}\left(M^{q}\right) \cong 0$ and we conclude, using the usual Poincaré duality argument that $M^{\prime}$ is a homology 3-sphere.

In some recent work done by Culler, Gordon, Luecke, and Shalen, it is shown that if $(1, q)$-surgery on a nontrivial knot yields a simply connected manifold then $|q|=0$ or $1[\mathbf{C}, \mathbf{G}, \mathbf{L}, \mathbf{S}]$. If a knot $\Sigma$ has group $K$ and $m, l \in K$ is the meridian and the longitude, then the fundamental group of the surgery manifold $\Sigma(1, q)$ is $K /\left\langle\left\langle m l^{q}\right\rangle\right\rangle$. We thus have the following proposition. 
Proposition 4.3. If $K$ is a classical knot group and $q>1$ then $K /\left\langle\left\langle m l^{q}\right\rangle\right\rangle \neq 1$.

THEOREM 4.4. Let $K$ be a classical knot group with meridian $\mu$ and let $G=K \underset{\mu=t^{q}}{*}\langle t\rangle, q>1$. Then $G$ is not a classical knot group.

Proof. Let $M=X \cup_{A} T^{3}$ be the cabled manifold we constructed in the paragraph preceding Proposition 4.2 , then $\pi_{1}(M) \cong G$. Suppose there exists a knot $L$ in $S^{3}$ with $\pi_{1}\left(S^{3}-L\right)=G$. We shall eventually show that $L$ must be a cable knot about some core $L^{\prime}$ and that the surgery manifold $L^{\prime}(1, q)$ is simply connected which contradicts Proposition 4.3.

The knot manifold $X$ is aspherical [P]. Moreover, $M$ as the union of aspherical spaces sewn together along an incompressible subspace is aspherical $\left[\mathbf{W}_{2}\right]$. Let $N$ be the knot manifold for $L$. Since $\pi_{1}(M)$ is isomorphic to $\pi_{1}(N)$ and $M$ and $N$ are aspherical spaces, there exists a homotopy equivalence $f: N \rightarrow M$ that induces the group isomorphism.

The annulus $A$ is bicollared in $M$. Furthermore, $\pi_{1}(A) \rightarrow \pi_{1}(M)$ is injective since $\pi_{1}(A) \rightarrow \pi_{1}(X)$ and $\pi_{1}(A) \rightarrow \pi_{1}\left(T^{3}\right)$ are injective; also $\pi_{2}(A)=\pi_{2}(M)=\pi_{3}(M)=\pi_{2}(M-A)=0$ since $M, X$ and $T^{3}$ are aspherical spaces. Hence $\operatorname{ker}\left(\pi_{j}(A) \rightarrow \pi_{j}(M)\right)=0, j=1,2$. By Lemma 1.1 in $\left[\mathbf{W}_{1}\right]$ there exists a map $g$ that is homotopic to $f$ such that:

1. $g$ is transverse with respect to $A$, i.e. there exists a neighbourhood $g^{-1}(A) \times I$ of $g^{-1}(A)$ so that $g(x, y)=(g(x), y)$ for every $x \in$ $g^{-1}(A)$ and $y \in I$.

2. $g^{-1}(A)$ is an orientable compact 2-manifold and $g^{-1}(A) \cap \operatorname{Bd}(M)=$ $\operatorname{Bd}\left(g^{-1}(A)\right)$.

3. If $F$ is a component of $g^{-1}(A)$ then $\operatorname{ker}\left(\pi_{j}(F) \rightarrow \pi_{j}(N)\right)=0, j=$ 1,2 .

Choose a map $g$ that minimizes the number of components of $g^{-1}(A)$. We shall show that for such a map $g, g^{-1}(A)$ is just one annulus $F$ and that $F$ separates $N$ into a solid torus $V$ and a knot manifold $Y$ having the same group as $X$. We use techniques similar to those used in $[\mathbf{F}, \mathbf{W}]$ and $[\mathbf{S}]$ to prove the following assertions. (Some proofs are omitted since they are the same as proofs in [F, W] and [S].)

Claim 4.4.1. $g^{-1}(A)$ is nonempty.

Claim 4.4.2. Let $F$ be a component of $g^{-1}(A)$. Then $F$ is an essential annulus. 
We thus have $g^{-1}(A)=F_{1} \cup \cdots \cup F_{K}, k \geq 1$. Since each component of $g^{-1}(A)$ is an essential annulus we get that the core $L$ of $S^{3}-\operatorname{Int}(N)$ is either a composite knot or a cable knot ([W $\left.\mathbf{W}_{3}\right]$, Lemma 1.1).

Claim 4.4.3. $L$ is a cable knot.

Since $g^{-1}(A)=F_{1} \cup \cdots \cup F_{K}$ and $L$ is a $\left(p^{\prime}, q^{\prime}\right)$-cable about a knot $L^{\prime}$ we have for each $i, 1 \leq i \leq k, N=Y_{i} \cup_{F_{i}} V_{i}$ where $V_{i}$ is a solid torus and $Y_{i}$ is a knot manifold. Moreover a boundary component of $\operatorname{Bd}\left(F_{i}\right)$ is a $\left(p^{\prime}, q^{\prime}\right)$-curve on $S^{3}-\operatorname{Int}\left(Y_{i}\right)$.

Claim 4.4.4. $\pi_{1}(N)$ has trivial center.

Proof of 4.4.4. Since $\pi_{1}(N)=K \underset{\mu=t^{q}}{*}\langle t\rangle$, the center of $\pi_{1}(N)=$ $C(K) \cap\langle\mu\rangle\left([\mathbf{M}, \mathbf{K}, \mathbf{S}]\right.$, Cor. 4.5). If $K t^{\mu}$ is not a torus knot group then $C(K)=1$ and consequently $\pi_{1}(N)$ has no center. On the other hand if $K$ is a torus knot group then $C(K)=\left\langle\mu^{p q} \lambda\right\rangle$, but $\left\langle\mu^{p q} \lambda\right\rangle \cap\langle\mu\rangle=1$ in $K$ and consequently in $K \underset{\mu=t^{q}}{*}\langle t\rangle$.

Claim 4.4.5. The annuli $F_{1}, \ldots, F_{K}$ are parallel in $N$.

Proof of 4.4.5. $N$ is prime since it is a cable knot manifold. Moreover $N$ is irreducible. Since $\pi_{1}(N)$ has trivial center, $N$ cannot be a Siefert fiber space with decomposition surface a disk with 3 singular fibers as such a space has fundamental group with nontrivial center [J]. By Lemma 2.4 in $[\mathbf{J}, \mathbf{M}]$ there exists a unique (up to ambient isotopy) essential annulus embedded in $N$. Hence each annulus $F_{i}$ is parallel to $F_{1}$.

Claim 4.4.6. For each $i, 1 \leq i \leq k,\left.g\right|_{F_{i}}$ is homotopic to a homeomorphism.

Proof of 4.4.6. It suffices to show that $g_{*}: H_{1}\left(F_{i}\right) \rightarrow H_{1}(A)$ is an isomorphism. Since $H_{1}\left(F_{i}\right) \cong \pi_{1}\left(F_{i}\right)$ and $H_{1}(A) \cong \pi_{1}(A)$, this implies that $g_{*}: \pi_{1}\left(F_{i}\right) \rightarrow \pi_{1}(A)$ is an isomorphism. Hence $\left.g\right|_{F_{i}}$ is a homotopy equivalence and is therefore homotopic to a homeomorphism.

Let $f_{i}$ be a generator for $H_{1}\left(F_{i}\right)$ and let $a$ be a generator for $H_{1}(A)$. Then $g_{*}\left(f_{i}\right)=a^{r}$. We wish to show that $r= \pm 1$. We use a homotopy inverse $h$ of $g(h: M \rightarrow N)$. As done earlier in the proof we can modify $h$ such that $h^{-1}\left(F_{i}\right)$ is a collection of essential annuli, $B_{1}, \ldots, B_{n}$. Since $M$ is irreducible ([J], Lemma 3.1) and $M$ is not a twisted $I$-bundle over the Klein bottle (if $M$ is a twisted $I$-bundle over the Klein bottle then $\pi_{1}(M)$ 
abelianizes to $\left.Z \oplus Z_{2}\right)$ there exists an isotopy $h_{t}(0 \leq t \leq 1)$ such that $h_{1}\left(\operatorname{Bd}\left(B_{i}\right)\right) \cap \operatorname{Bd}(A) \neq \varnothing$. Hence $\operatorname{Bd}\left(B_{i}\right)$ is homologous to $\operatorname{Bd}(A)$ in $H_{1}(M)$, i.e. the generator $a$ for $H_{1}(A)$ is homologous to a generator $b_{i}$ for $H_{1}\left(B_{i}\right)$. Furthermore, $h_{*}\left(b_{i}\right)=f_{i}^{s}$. Since $h$ is a homotopy inverse of $g$ we get $g_{*}\left(h_{*}\left(b_{i}\right)\right)=b_{i}$, i.e. $a^{s r}=b_{i}$. Since $a$ is homologous to $b_{i}$ in $H_{1}(M)$ we obtain $a^{s r}=a$ in $H_{1}(M)$. Hence $s r=1$, and it follows that $r= \pm 1$.

Claim 4.4.7. $p^{\prime} q^{\prime}=q$.

Proof of 4.4.7. $z_{p^{\prime} q^{\prime}} \cong H_{1}(N) / H_{1}\left(F_{i}\right) \cong H_{1}(M) / H_{1}(A) \cong Z_{q}$.

Recall that $g^{-1}(A)=F_{1} \cup \cdots \cup F_{K}$.

Claim 4.4.8. $k$ is odd.

Proof of 4.4.8. Since the annuli $F_{i}, 1 \leq i \leq k$ are all parallel $V_{k}$ contains a core $v_{1}$ of $V_{1}$. Let $\alpha$ be a path in $V_{1}$ from $F_{1}$ to $v_{1}$, then $\pi_{1}\left(Y_{1}\right)$ and $\alpha v_{1} \alpha^{-1}$ generate $\pi_{1}(N)$. If $k$ is even then $g$ maps $\operatorname{Int}\left(Y_{1}\right)$ and $v_{1}$ into the same component of $M-A$. If $Y_{1}$ and $v_{1}$ are both mapped into $X-A$, then $\pi_{1}(M) / \pi_{1}(X) \cong 1$ which contradicts the fact that $H_{1}(M) / H_{1}(X) \cong Z_{q}$. On the other hand $Y_{1}$ cannot be mapped into $T^{3}$, because that would imply that $g_{*}: \pi_{1}\left(Y_{1}\right) \rightarrow \pi_{1}\left(T^{3}\right)$ is $1-1$ which contradicts the fact that $Y_{1}$ is a knot manifold.

Claim 4.4.9. $k=1$.

The proof of 4.4.9 is the same as the proof of Claim 7 in [S].

Since $k=1$, we have that $N=Y \cup_{F} V$, where $Y$ is the knot manifold for the knot $L^{\prime}, V$ is a solid torus, and a boundary component of $\operatorname{Bd}(F)$ is a $\left(p^{\prime}, q^{\prime}\right)$-curve of the boundary of $S^{3}-\operatorname{Int}(Y)$. Moreover we have a homotopy equivalence $g: Y \cup_{F} V \rightarrow X \cup_{A} T^{3}$ such that $g_{\mid F}$ is a homeomorphism. We saw in the proof of Claim 4.4.8 that $g(Y)$ is not contained in $T^{3}$. Hence $g(Y) \subseteq X$ and $g(V) \subseteq T^{3}$ and $g_{*}\left(\pi_{1}(Y)\right) \subset \pi_{1}(X)$ and $g_{*}\left(\pi_{1}(V)\right) \subseteq \pi_{1}\left(T^{3}\right)$. Let $s$ be a generator for $\pi_{1}(V)$ and let $m, l$ be a meridian, longitude pair for $\pi_{1}(Y)$, then $\pi_{1}(N)=\pi_{1}(Y) * \pi_{1}(F) \pi_{1}(V)=$

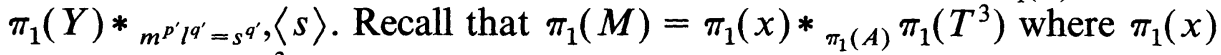
$=K$ and $\pi_{1}\left(T^{3}\right)=\langle t\rangle$. Since $g_{*}$ is an isomorphism and $g_{*}\left(\pi_{1}(F)\right)=$ $\pi_{1}(A)$ we have the following $g_{*}\left(\pi_{1}(N)\right)=g_{*}\left(\pi_{1}(Y)\right){ }_{\pi_{1}(A)} g_{*}\left(\pi_{1}(V)\right)=$ $K * \pi_{\pi_{1}(A)}\langle t\rangle$. By Proposition 2.5 in [B] we conclude that $\left.g_{*}\right|_{\pi_{1}(Y)}: \pi_{1}(Y) \rightarrow$ $K$ and $\left.g_{*}\right|_{\pi_{1}(V)}: \pi_{1}(V) \rightarrow \pi_{1}\left(T^{3}\right)$ is an isomorphism. Therefore, since 
$q=p^{\prime} q^{\prime}$ we have the following: $g_{*}\left(\left(m^{p^{\prime}} l^{q^{\prime}}\right)^{p^{\prime}}\right)=g_{*}\left(s^{q^{\prime} p^{\prime}}\right)=g_{*}\left(s^{q}\right)=t^{ \pm q}$ $=\mu^{ \pm 1}$. Hence $\pi_{1}(Y) /\left\langle\left\langle\left(m^{p^{\prime}} l^{q^{\prime}}\right)^{p^{\prime}}\right\rangle\right\rangle \cong K /\left\langle\left\langle\mu^{ \pm 1}\right\rangle\right\rangle=1$. Since $\pi_{1}(Y) /\left\langle\left\langle\left(m^{p^{\prime}} l^{q^{\prime}}\right)^{p^{\prime}}\right\rangle\right\rangle$ abelianizes to $Z_{\left(p^{\prime}\right)^{2}}$ this implies that $p^{\prime}= \pm 1$ which in turn implies that $q^{\prime}= \pm q$. Hence $\pi_{1}(Y) /\left\langle\left\langle m l^{q}\right\rangle\right\rangle=1$ which contradicts Proposition 4.3.

\section{REFERENCES}

[B] E. M. Brown, Unknotting in $M^{2} \times I$, Trans. Amer. Math. Soc., 123 (1966), 480-505.

[C, G, L, S] M. Culler, C. McA. Gordon, J. Luecke, and P. Shalen, Dehn surgery on knots, Bull. Amer. Math. Soc., 13, no. 1 (1985), 43-45.

[F, W] C. D. Feustel, and W. Whitten, Groups and complements of knots, Canad. J. Math., 30, no. 6 (1978), 1284-1295.

[F] R. H. Fox, A quick trip through knot theory, in Topology of 3-Manifolds and Related Topics, M. K. Fort, Ed., Prentice Hall (1966), 120-167.

[F, M] R. H. Fox and J. W. Milnor, Singularities of 2-spheres in 4-space and cobordism of knots, Osaka J. Math., 3 (1966), 257-267.

[J] W. Jaco, Lectures on three-manifold topology, Conference Board of the Mathematical Sciences by the Amer. Math. Soc. no. 43, (1977).

[J, M] W. Jaco and R. Meyers, An algebraic determination of closed orientable 3-manifolds, Trans. Amer. Math. Soc., 253 (1979), 149-170.

[K] M. Kervaire, On higher dimensional knots, in Differential and Combinatorial Topology, S. S. Cairns, Ed., Princeton University Press.

[M, K, S] W. Magnus, A. Karrass and D. Solitar, Combinatorial Group Theory, (2nd revised ed.), Dover Pub. Inc., New York (1966).

[P] C. D. Papakyriakopoulos, On Dehn's Lemma and the asphericity of knots, Proc. Nat. Acad. Sci., 43 (1957), 169-172.

$\left[\mathrm{R}_{1}\right] \quad$ J. G. Ratcliffe, $A$ fibered knot in a homology 3-sphere whose group is non-classical, Contemporary Math., 20 (1983).

$\left[\mathrm{R}_{2}\right] \quad$ D. Rolfsen, Knots and Links, Publish or Perish Inc. (1976).

[S] J. K. Simon, An algebraic classification of knots in $S^{3}$, Ann. of Math., (2) 97 (1973), 1-13.

$\left[\mathrm{W}_{1}\right] \quad \mathrm{F}$. Waldhausen, Gruppen mit zentrum und 3-dimensionale mannigfaltigeiten, Topology, 6 (1967), 505-517.

$\left[\mathrm{W}_{2}\right] \quad$ J. H. C. Whitehead, On the asphericity of regions in a 3-sphere, Fund. Math., 32 (1939), 259-270.

$\left[\mathrm{W}_{3}\right] \quad \mathrm{W}$. Whitten, Algebraic and geometric characterizations of knots, Invent. Math., 26 (1974), 259-270.

Received June 1, 1986 and in revised form September 8, 1986.

College of Charleston

Charleston, SC 29424

Current address: Department of Mathematics

California State University, Northridge

Northridge, CA 91330 



\section{PACIFIC JOURNAL OF MATHEMATICS EDITORS}

V. S. VARADARAJAN (Managing Editor) University of California Los Angeles, CA 90024 HERBERT ClEMENS University of Utah Salt Lake City, UT 84112

R. FINN

Stanford University

Stanford, CA 94305

\section{HERMANN FLASCHKA} University of Arizona Tucson, AZ 85721

RAMEsh A. GANGolli University of Washington Seattle, WA 98195

VAUghan F. R. JONES

University of California Berkeley, CA 94720

\section{ROBION KIRBY}

University of California

Berkeley, CA 94720

C. C. MOORE

University of California

Berkeley, CA 94720

HAROLD STARK

University of California, San Diego

La Jolla, CA 92093

\section{ASSOCIATE EDITORS}
R. ARENS
E. F. BECKENBACH
B. H. NEUMANN
F. WOLF
K. YOSHIDA (1906-1982)

\section{SUPPORTING INSTITUTIONS}

UNIVERSITY OF ARIZONA

UNIVERSITY OF BRITISH COLUMBIA

CALIFORNIA INSTITUTE OF TECHNOLOGY

UNIVERSITY OF CALIFORNIA

MONTANA STATE UNIVERSITY

UNIVERSITY OF NEVADA, RENO

NEW MEXICO STATE UNIVERSITY

OREGON STATE UNIVERSITY
UNIVERSITY OF OREGON

UNIVERSITY OF SOUTHERN CALIFORNIA

STANFORD UNIVERSITY

UNIVERSITY OF HAWAII

UNIVERSITY OF TOKYO

UNIVERSITY OF UTAH

WASHINGTON STATE UNIVERSITY

UNIVERSITY OF WASHINGTON 


\section{Pacific Journal of Mathematics}

Vol. 130, No. $1 \quad$ September, 1987

K. Adachi, Continuation of bounded holomorphic functions from certain subvarieties to weakly pseudoconvex domains $\ldots \ldots \ldots \ldots \ldots \ldots \ldots$

Erazm Jerzy Behr, Enveloping algebras of Lie superalgebras ........... 9

Dong M. Chung, Scale-invariant measurability in abstract Wiener spaces . . . 27

Peter Gerard Dodds and Bernardus de Pagter, Algebras of unbounded

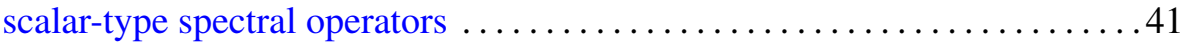

Wu-Yi Hsiang and Hsueh-Ling Huynh, Generalized rotational

hypersurfaces of constant mean curvature in the Euclidean spaces. II . . . .75

Harvey Bayard Keynes and M. Sears, Time changes for $\mathbf{R}^{n}$ flows and suspensions ..................................... 97

Frances Kirwan, Ronnie Lee and Steven Howard Weintraub, Quotients

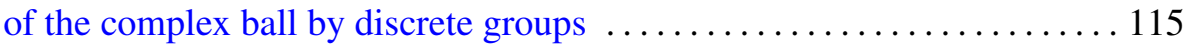

Magnhild Lien, Groups of knots in homology 3-spheres that are not

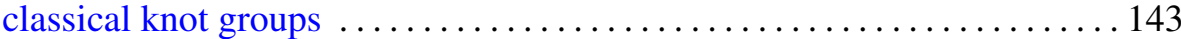

Juan Carlos Migliore, Liaison of a union of skew lines in $\mathbf{P}^{4} \ldots \ldots \ldots \ldots 153$

Jesper M. Møller, Spaces of sections of Eilenberg-Mac Lane fibrations . . . . 171 Daniel Ullman, A generalization of a theorem of Atkinson to noninvariant

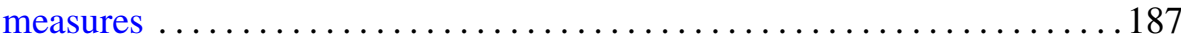

Kohhei Yamaguchi, Operations which detect $\mathscr{P}^{1}$ in odd primary connective

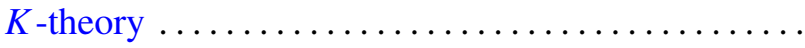

